\title{
Role of mHealth in getting Target of Universal Health Coverage
}

\author{
Khalida Naz Memon
}

This editorial may be cited as: Memon KN. Role of mHealth in getting Target of Universal Health Coverage. J Liaquat Uni Med Health Sci. 2019;18(02):82-3. doi: 10.22442/jlumhs.191820605

Universal Health Coverage (UHC)is firmly based on the WHO constitution of 1948 wherein health was declared as a fundamental human right and on the Health for All (HFA) agenda set by the Alma Ata declaration in 1978. The concept of UHC cuts across all of the health-related Sustainable Development Goals (SDGs) and brings hope of better health and protection for the world's poorest community. It implies that all people should have access to healthcare, meaning thereby that all people and communities can use the promotive, preventive, curative, rehabilitative and palliative health services they need; these health services must be of sufficient quality, effective and must also ensuring that the use of these services does not expose the user to financial hardship.

In essence, it fulfills three main objectives in health delivery for common people. First it ensures equity in access to health services i.e. everyone who needs services should get them, not only those who can pay for them. Second the quality of health services should be good enough to improve the health of those receiving services; and third, ensures a sound financing system to protect people from financial hardship and impoverishment from health care costs.

The World Health Organization (WHO) has published its draft on Global Strategy for Digital Health, which puts digital health initiatives and greater use of technology and health information systems at the heart of achieving affordable and universal access to health care.

Achieving UHC by 2030 will only be possible if a collaborative ecosystem is created that makes the public and private health sectors as a major part of the digital transformation. Ongoing digitalization and the introduction of new technologies, like telehealth, are already creating patient-centered healthcare systems having greater potential to improve patient outcomes and the efficiency of health care delivery. mHealth (mobile health) is a medical and public health practice supported by mobile devices, such as cell phones, patient monitoring devices, personal digital assistants (PDAs), and other wireless devices.
Mobile phone usage has been rapidly increasing worldwide. The popularity of mobile devices in the developed or developing world presents the opportunity to improve health outcomes through the delivery of innovative medical and health services with information and communication technologies (ICT) to the farthest corners of the globe. With this background, mHealth has been a priority for the World Health Organization (WHO) since 2005. It was resolved that mHealth effectively improves the quality of care and that it can quickly be adopted on a large scale, at low cost and in secured manner. The ICT can support health and health related fields, including health-care services, health surveillance, health literature, and health education, knowledge and research. To help achieve overarching health priorities such as UHC, mHealth strategies are needed which can support resilient health systems.

Practically speaking, mHealth can take the shape of a quick communication between health services and individuals pertaining to treatment adherence through reminder messages provided by health services to patients aiming at achieving medication adherence using mobile ICT; messages can be text, voice or multimedia. It can also be in the form of reminders to attend appointments. mHealth can also help in community mobilization/health promotion campaigns especially to raise health awareness among target groups. It can also take the shape of consultation between health care practitioners. Intersectoral communication \& health management system facilitation in situations of health emergencies/ disasters is another proposed avenue for mHealth. It can also help health authorities in health monitoring and surveillance, health surveys, data collection, management and reporting of health surveys using mobile ICT. Besides this, mHealth can also help clinical decisions by facilitating access to electronic patient information \& decision support systems using mobile ICT.

mHealth can efficiently deliver high-quality universal health care, but the evidence supporting its current 
effectiveness in our country is still mixed. What's needed to foster mHealth growth in the developing world is advocacy \& a thoughtful policy. How best to grasp the opportunity to work in a cross-sector way to monitor and manage the broader determinants of health, remains to be explored. In doing so, it should explore how a patient-centred health service can be supported by cross-sector work, and thereby lay the $\mathrm{mHealth}$ foundations to attain the target of universal health coverage by 2030 .

AUTHOR AFFILIATION:

Prof. Khalida Naz Memon

Department of Community Medicine

Liaquat University of Medical and Health Sciences

Jamshoro, Sindh-Pakistan.

Email: knazmemon63@hotmail.com 